\title{
Norois
}

Environnement, aménagement, société

\section{La démarche de développement d'indicateurs basés sur l'ichtyofaune pour évaluer la qualité écologique des eaux de transition françaises dans le cadre de la DCE : une marche forcée pour des résultats positifs}

Developing a fish-based indicator to estimate the ecological status of transitional water in france for the Water Framework Directive: a fast but successful approach

Olivier Le Pape, Mario Lepage et Philippe Féra

\section{OpenEdition}

Journals

Édition électronique

URL : http://journals.openedition.org/norois/5632

DOl : $10.4000 /$ norois. 5632

ISBN : 978-2-7535-4775-9

ISSN : $1760-8546$

Éditeur

Presses universitaires de Rennes

Édition imprimée

Date de publication : 15 novembre 2015

Pagination : 37-49

ISBN : 978-2-7535-4771-1

ISSN : 0029-182X

Référence électronique

Olivier Le Pape, Mario Lepage et Philippe Féra, «La démarche de développement d'indicateurs basés sur l'ichtyofaune pour évaluer la qualité écologique des eaux de transition françaises dans le cadre de la DCE : une marche forcée pour des résultats positifs », Norois [En ligne], 235 | 2015, mis en ligne le 15 novembre 2017, consulté le 19 avril 2019. URL : http://journals.openedition.org/norois/5632 ; DOI : 10.4000/norois.5632 


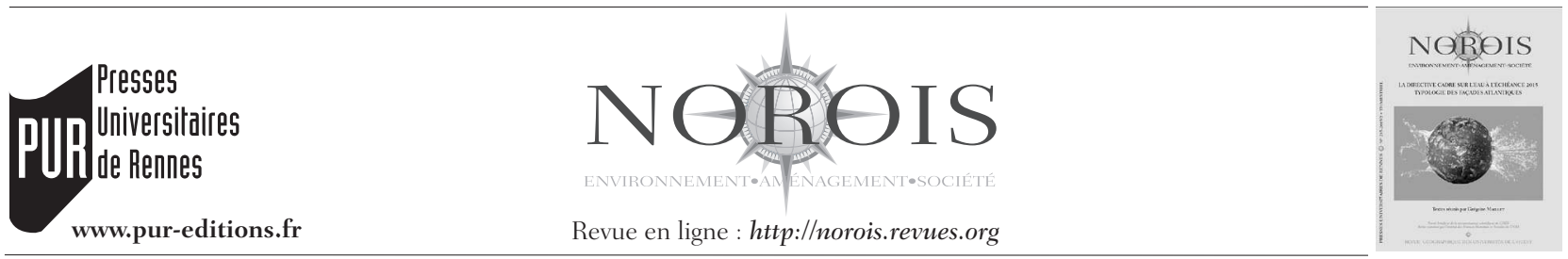

\title{
La démarche de développement d'indicateurs basés sur l'ichtyofaune pour évaluer la qualité écologique des eaux de transition françaises dans le cadre de la DCE : une marche forcée pour des résultats positifs
}

\author{
Developing a Fish-based Indicator to Estimate the Ecological Status of Transitional Water \\ in France for the Water Framework Directive: A Fast but Successful Approach
}

\author{
Olivier Le PAPE ${ }^{\mathrm{a}^{*}}$, Mario LePage ${ }^{\mathrm{b}}$ et Philippe FÉrA ${ }^{\mathrm{c}}$
}

\footnotetext{
*Auteur correspondant

a UMR 985 ESE (INRA-Agrocampus-Ouest), 65 rue de Saint-Brieuc, CS 8421535042 Rennes Cedex, France. (lepape@agrocampus-ouest.fr)

${ }^{\mathrm{b}}$ Irstea, UR EPBX - 50 av. de Verdun, F-33612 Cestas, France.

cAgence de L'eau Loire Bretagne, Délégation Ouest-Atlantique - 1 rue Eugène-Varlin, CS 40521, 44105 NANTES

Cedex 4, France.
}

Résumé : Cet article se propose d'expliquer, sur l'exemple de l'ichtyofaune des masses d'eau de transition, la démarche adoptée par la France pour répondre aux objectifs de la DCE et les défis qu'elle présentait dans des délais contraints. Nous présentons le vide de connaissances et de données qui régnait au début des années 2000 et les actions mises en œuvre au pas de charge pour répondre aux objectifs ambitieux de la DCE, disposer d'un réseau de surveillance en 2007, puis d'indicateurs normés décrivant l'état écologique afin de présenter un bilan six ans plus tard, en 2013, à l'issue du premier plan de gestion. Il reste désormais à poursuivre la démarche en milieu marin dans le cadre de la DCSMM.

Abstract: In this paper, we propose to study fish in transition waters to illustrate the challenge of meeting the goals of the European Union Water Framework Directive (EWFD) in France in restricted delays. We present the lack of knowledge and data in early 2000 s and the actions led then to achieve the ambitious objectives of the EWFD: the creation of a standardized monitoring network in 2007 and the definition of ecological indicators based on the fish community. In 2013, at the end of the first management plan, an assessment of the ecological status was presented. These actions have now to be continued towards marine waters in relation with the EU Marine Strategy Framework Directive.

Mots clés : DCE - indicateur - poisson - estuaire - gestion des ressources en eau - Union européenne

Keywords: EWFD, indicators, fish, estuary, water management, European Union 


\section{INTRODUCTION}

Lors de sa publication en octobre 2000, la Directive Cadre sur l'Eau (DCE), qui a pour objectif l'atteinte du bon état physico-chimique et écologique des eaux européennes en 2015, représentait un défi majeur pour les milieux côtiers et les secteurs de transition que constituent les estuaires et les lagunes littorales. En effet, bien que le caractère dynamisant de cette directive pour reconquérir la qualité des eaux en Europe s'applique à l'ensemble des catégories de masses d'eaux, elle ne présentait pas en 2000 les mêmes enjeux en termes de connaissance, de mise en place de réseaux de surveillance, d'indicateurs disponibles et de gestion pour les milieux en interactions avec la mer que pour les systèmes aquatiques continentaux (cours d'eau, plan d'eau et eaux souterraines).

Les politiques européennes s'étaient attachées depuis les années 1970 à instaurer des textes réglementaires sectoriels et normatifs portant soit sur les usages (eau potable, baignades, eaux conchylicoles, etc.) soit sur les rejets (substances dangereuses, eaux résiduaires urbaines, nitrates, etc.). La grande nouveauté introduite par la DCE, outre son aspect d'encadrement des textes existants, a été d'introduire les notions de qualité écologique quantifiable et d'obligation de résultats. Ce défi était d'autant plus grand pour les eaux côtières et de transition par rapport aux eaux continentales que les données et les indicateurs biologiques disponibles étaient rares, voire inexistants.

Depuis le début des années 1980, les eaux continentales bénéficiaient de grilles de qualité physicochimiques des eaux et d'indices biologiques basés sur la présence ou non d'organismes polluo-sensibles (e.g. l'ichtyofaune; Oberdorff et al., 2002; Pont et al., 2007; Roset et al., 2007). Ces indicateurs ont ensuite été confortés dans les années 1990 par la mise en place d'un Système d'Évaluation de Qualité (SEQ - Eau). En conséquence, la DCE consistait pour les eaux continentales en une étape supplémentaire d'un processus initié depuis une vingtaine d'années pour aller plus avant vers une qualification de l'état écologique et opérer une harmonisation au sein d'une directive unique.

En revanche, pour les eaux marines, l'appréciation de la qualité des eaux et des milieux préalablement à la DCE se faisait surtout en référence à la qualité sanitaire des usages baignade (1975) et conchyliculture (1979), sur un nombre limité de secteurs concernés par ces usages. L'appréciation de la qualité chimique des milieux côtiers a débuté en 1974 par la mise en place du Réseau National d'Observation ( $\mathrm{RNO}$, désormais ROCCH) par Ifremer. Pour autant, devant le manque de normes ou de références de qualité, ce réseau qualifiait régulièrement les sites côtiers français en les comparants entre eux et par rapport à la moyenne nationale (Claisse, 1989). Il existait bien une grille d'analyse, mais celle-ci était adaptée à la qualification de sédiments pollués et non en référence à des teneurs naturelles. Les premiers réseaux biologiques marins pérennes ont vu le jour en 1984 pour le réseau phytoplanctonique REPHY, en 1997 pour le réseau « ulves » en Bretagne et en 2003 pour le réseau benthique Rebent Bretagne, suite à l'échouage du pétrolier Erika, là aussi sur un nombre limité de secteurs (REPHY en zone conchylicole, «Ulves » sur les sites impactés en Bretagne et Rebent sur un nombre restreint de sites pilotes). Ces réseaux se limitaient, par manque de normes ou de références, à comparer les sites entre eux ou de façon interannuelle. C'est vraiment la mise en œuvre de la DCE, avec l'introduction de la notion de bon état et l'obligation de créer des indicateurs, qui a permis le développement de métriques et de grilles de qualité pour les eaux côtières et de transition. Tout restait donc à mettre en place pour ces milieux à l'édition de la DCE en octobre 2000.

La DCE spécifie que la surveillance écologique se base sur les compartiments biologiques suivants : le phytoplancton, la flore aquatique, la faune benthique invertébrée pour les eaux côtières, les mêmes compartiments plus l'ichtyofaune pour les eaux de transition. La première partie de la décennie 2000 a donc consisté en une course contre la montre pour développer des protocoles et mettre en place des indicateurs et des normes de qualité, afin de démarrer le programme de surveillance en 2007, conformément à la DCE. En se basant sur des réseaux opérationnels existants (en adaptant le réseau phytoplancton et phycotoxines pour le cas du phytoplancton) ou en développant, ex-nihilo ou presque, de nouveaux réseaux de surveillance (le Rebent initié en 2000 pour les invertébrés benthiques), il a fallu combler le retard structurel de plusieurs décennies pris sur ces milieux par rapport aux eaux continen- 
tales pour répondre aux exigences de la DCE dans les mêmes délais. Ce défi se doublait de problèmes spécifiques aux eaux côtières et de transition. En effet ces milieux fluctuants, et notamment les estuaires, présentent des communautés écologiques adaptées aux gradients spatio-temporels rapides des conditions environnementales (e.g. la salinité). En conséquence, leurs caractéristiques intrinsèques se rapprochent de celles des communautés de milieux perturbés (estuarine quality paradox; Elliott et Quintino, 2007). La mise en évidence de l'effet de pressions anthropiques et la qualification du bon état écologique y est donc plus délicate qu'ailleurs.

Cet article se propose d'expliquer, sur l'exemple de l'ichtyofaune estuarienne, le vide qui régnait au début des années 2000 et les démarches mises en œuvre au pas de charge pour répondre aux objectifs ambitieux de la DCE, disposer d'un réseau de surveillance en 2007, puis d'indicateurs normés décrivant l'état écologique afin de présenter un premier bilan six ans plus tard, en 2013, à l'issue du premier plan de gestion. La première partie présente les objectifs fixés par la DCE. Les deux parties suivantes décrivent le travail réalisé pour la France métropolitaine en ce qui concerne l'ichtyofaune des estuaires, avec de brefs compléments pour les lagunes. La dernièer partie évoque l'harmonisation européenne réalisée sur la base des stratégies nationales.

\section{Suivi de la qualité ÉCOLOGIQUE DANS LES EAUX DE TRANSITION DANS LE CADRE DE LA DCE : PRÉCONISATIONS, DÉMARCHE ET TRAVAUX PRÉLIMINAIRES}

Une notion nouvelle apportée par la DCE est la notion de «Masse d'eau ». La DCE demande aux états membres d'établir un inventaire de masses d'eau regroupées par types homogènes. Une masse d'eau représente une unité hydraulique homogène dont la qualité peut, d'une part, être estimée par un nombre limité de point de contrôle, et d'autre part, être rapportée auprès de l'union européenne. Un travail préliminaire à l'échelle nationale, impliquant de nombreux acteurs, a donc consisté à délimiter les masses d'eau côtières et de transition sur la base de critères hydrologiques (courant, temps de résidence, stratification, etc.) et de nature des fonds.
Spécifiquement, en ce qui concerne les estuaires, la DCE précise que la zone couverte par chaque masse d'eau estuarienne s'étend de la limite amont de la marée dynamique jusqu'à une limite aval qui sépare les eaux estuariennes et côtières, limite à définir pour chaque site. La délimitation spatiale des masses d'eau de type lagune, l'autre type de masses d'eau de transition, semble plus naturelle mais des choix de gestion ont conduit à agréger plusieurs lagunes pour constituer une masse d'eau.

La DCE demande aux états membres de suivre un certain nombre des masses d'eau inventoriées, en prenant en compte tous les éléments de qualité biologique (i.e. phytoplancton, flore, invertébrés benthiques et poissons pour les eaux de transition). L'article 8 de la DCE prévoit la mise en œuvre d'un programme de surveillance, de manière à « dresser un tableau cohérent et complet de l'état des eaux au sein de chaque bassin hydrographique ». Ce programme est mené sur la durée d'un «plan de gestion », soit 6 ans. Le programme de surveillance comprend quatre types de contrôles :

- le contrôle de surveillance, porte sur tous les paramètres biologiques et chimiques. Il a vocation à s'exercer sur un nombre représentatif de masses d'eau pour permettre une évaluation générale de l'état écologique et chimique des eaux à l'échelle du bassin hydrographique;

- le contrôle opérationnel, mis en place sur les masses d'eau à risque de non-respect des objectifs environnementaux (RNROE), porte sur les paramètres responsables de la mauvaise qualité des masses d'eau;

- le contrôle d'enquête, mis en œuvre pour rechercher les causes d'une mauvaise qualité en l'absence de réseau opérationnel ou pour évaluer l'ampleur et l'incidence d'une pollution accidentelle;

- le contrôle additionnel, destiné à vérifier les pressions qui s'exercent sur les zones "protégées », c'est-à-dire les secteurs ou activités déjà soumis à une réglementation européenne (ex. : zones conchylicoles, Natura 2000, baignades).

Des groupes d'experts nationaux ont été créés pour chaque paramètre biologique (i.e. phytoplancton, flore, invertébrés benthiques et poissons pour les eaux de transition) avec comme objectif de concevoir les réseaux de surveillance et leurs modalités (engins de prélèvements, stratégie spatio-temporelle d'échantillonnage) puis de mettre au point 
les indicateur et les grilles de qualité permettant de qualifier les masses d'eau en 5 classes.

\section{SURVEILLANCE DE L'ICHTYOFAUNE : UN EXISTANT PLUS QUE LIMITÉ EN ESTUAIRE EN 2000, UN RÉSEAU DE SURVEILLANCE À CONSTRUIRE EX NIHILO}

En ce qui concerne les communautés de poissons dans les eaux de transition, la DCE requiert de se baser sur la composition et l'abondance pour contribuer à définir le bon état écologique de ces masses d'eau. La construction de l'indicateur "Poissons» en eau de transition a été confiée à l'Irstea (Institut national de recherche en sciences et technologies pour l'environnement et l'agriculture) de Bordeaux. Cette partie présente comment un constat initial d'absence de données opérationnelles a conduit à mettre en place au pas de charge un réseau de surveillance totalement nouveau.

\section{Inventaire initial des données disponibles : un constat sans appel de grand vide}

L'Irstea a tout d'abord réalisé, en 2005, un inventaire des données existantes sur les communautés de poissons en estuaire. Bien qu'exhaustif, ce travail a été réalisé rapidement, d'autant plus facilement que le constat a été sans appel. Hormis les campagnes menées en Gironde, qui ne couvraient d'ailleurs pas l'ensemble du secteur estuarien (Lobry et al., 2003), et quelques inventaires anciens et partiels par ailleurs, aucun estuaire français ne faisait avant 2005 l'objet d'un suivi récurrent de son ichtyofaune. Le travail à réaliser partait donc de zéro et le constat était semblable pour les lagunes malgré une littérature un peu plus abondante.

\section{Mise en place rapide d'un réseau de surveillance entièrement nouveau}

Préalablement à la mise en place d'un réseau de surveillance des estuaires, un projet de recherche du programme LITEAU (action de recherche du ministère en charge du développement durable pour soutenir le développement de connaissances, méthodes et pratiques scientifiques utiles à la défi- nition et à la mise en œuvre d'actions collectives et de politiques publiques en mer et sur les littoraux dans une optique de développement durable) intitulé « Développement pour la DCE d'un indicateur multimétrique de la qualité des eaux de transition à partir de leurs peuplements de poissons " a été mené à bien. Ce projet réalisé en collaboration avec les Agences de l'eau des façades maritimes françaises, a permis l'acquisition de données sur 27 estuaires en Manche et Atlantique plus le grand Rhône et 12 lagunes méditerranéennes. À l'issue de cette phase de travail menée au milieu des années 2000, un protocole de suivi standardisé et applicable dans la majorité des estuaires français a été proposé en 2007 par l'Irstea ainsi qu'un protocole adapté au suivi des lagunes.

Ce travail a commencé par la mise au point d'une classification plus simple des masses d'eau, sur la base de la typologie française initiale en 9 types. In fine, et après des analyses et des regroupements successifs, les estuaires ont été distingués des lagunes, et séparés en deux classes de taille, les grands estuaires et les petits, et en deux écorégions, Manche et Atlantique. En raison d'enjeux de gestion particuliers à certaines zones, certains grands estuaires ont fait l'objet d'une stratification entre leurs parties fluviales, centrales et avals. Deux types ont été définis pour les lagunes contrairement à la typologie nationale qui n'en compte qu'un seul. C'est sur la base de cette classification simplifiée que se sont finalement construits les protocoles d'échantillonnage.

À l'issue d'un processus itératif intégrant des considérations financières, des échanges avec les personnes expertes en charge des prélèvements sur les différents estuaires et des retours de l'analyse des données et du travail de développement d'indicateurs fiables et normés sur la base de ces échantillonnages, les protocoles se sont affinés progressivement.

Dès le départ, pour des raisons de mise en pratique in situ, deux chaluts à perche (engin trainant sur le fond constitué d'un filet en forme de poche s'ouvrant par une perche horizontale et deux patins aux extrémités, tiré par un navire, figure 1) différents ont été utilisés dans les estuaires. Dans les grands estuaires il s'agit d'un chalut de 3 mètres de large, efficace même sur des fonds relativement profond $(>10 \mathrm{~m})$, alors qu'un chalut à perche de 
1,5 m, manipulable à partir de petites embarcations, est utilisé dans les petits estuaires. Un filet verveux jumeaux (engin fixe constitué de deux nasses en filet reliées par un filet droit jouant le rôle de guide) a été initialement utilisé en complément (Lepage et Girardin, 2006). L'utilisation de ce filet a finalement été abandonnée du fait de l'impossibilité de définir, à coût abordable en moyens humains et matériels, un protocole standardisé permettant une évaluation de l'ichtyofaune des petits fonds par ce biais.

Le protocole fixe la vitesse de chalutage entre 1,5 et 3 nœuds ( 1 nœud $=1852 \mathrm{~m} / \mathrm{h}$ ) selon le chalut, et la durée de chaque trait est fixée à 15 minutes. Si ces paramètres ont peu évolué, la couverture spatiale des masses d'eau a par contre fait l'objet d'ajustements. L'échantillonnage nécessitait initialement de couvrir l'ensemble de l'estuaire, de la limite amont à l'aval de la masse d'eau, en réalisant environ 25 traits de chaluts sur une campagne. Les premières analyses des données d'ichtyofaune ont confirmé que le gradient de salinité, depuis les eaux douce en amont jusqu'aux eaux marines de la limite aval, est très structurant pour les communautés de poissons (Courrat et al., 2009; Nicolas et al., 2010b). En conséquence, pour les estuaires au sein desquels on peut observer ce gradient (i.e. ce n'est pas systématiquement le cas des grands estuaires stratifiés en masses d'eau distinctes, ni des petits estuaires dont la partie dessalée se résume souvent à un tronçon fluvial court et étroit, notamment en période d'étiage), un minimum de 8 traits de chalut par classe de salinité (oligohalin : $\leq 5$ PSS, mesohalin : ]5,18] PSS, polyhalin : >18PSS) a été imposé. Un minimum de 12 traits de chalut est par contre fixé dans le cas où une masse d'eau comporte une seule zone de salinité (cas de l'estuaire de la Bidassoa ou de l'estuaire de la Seudre par exemple).

Une démarche similaire a permis de mettre en place un protocole d'échantillonnage de l'ichtyofaune en lagune, basé sur l'utilisation de capetchades (engins proches des filets verveux utilisés initialement en estuaire) posées à partir de la berge et laissé durant 4 jours avec une relève quotidienne (Lepage et Girardin, 2006).

Ce protocole de surveillance s'est mis en place et a été affiné progressivement à partir des premières campagnes réalisées en 2005. Fin 2012, près de 280 campagnes (échantillonnage d'une masse d'eau au cours d'une saison) avaient été réalisées dans

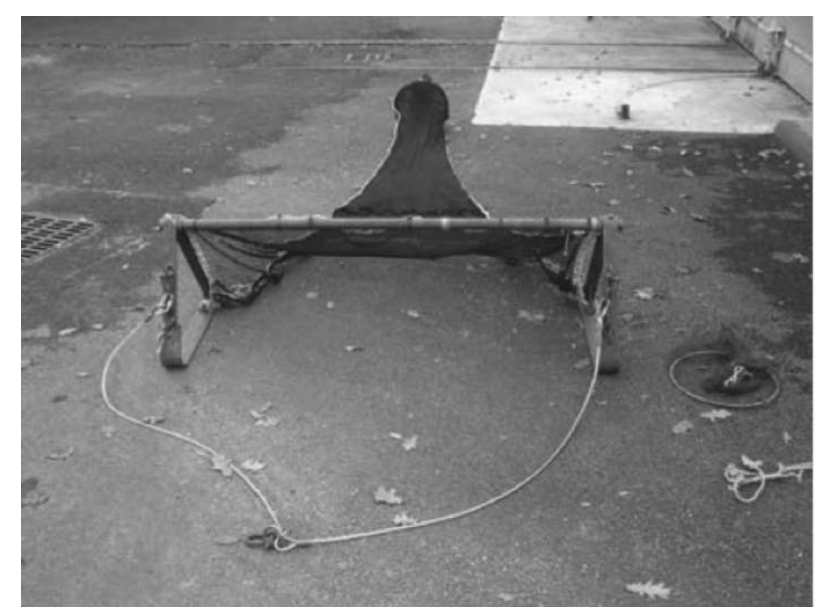

Figure 1 : Petit chalut à perche utilisé pour les échantillonnages d'ichtyofaune

Small beam trawl used for first fauna sampling

51 masses d'eau de transition françaises, 225 de ces campagnes concernaient 38 masses d'eau estuariennes, les autres portant sur 13 lagunes méditerranéennes.

\section{UNE MÉTHODE D'ANALYSE ET DES INDICATEURS BASÉS SUR L'ICHTYOFAUNE À DÉVELOPPER EX NIHILO, DES NORMES À FIXER}

Sur la base des données collectées lors des campagnes d'inventaire et de surveillance, la seconde phase du travail, démarrée lors du projet LITEAU à l'issue de la seconde année d'échantillonnage, début 2007, a consisté à mettre au point des indicateurs de qualité écologique portant sur l'ichtyofaune estuarienne. Pour ce faire, les spécifications de la DCE demandaient de prendre en compte la composition et l'abondance de cette ichtyofaune, en indiquant que les indicateurs poissons doivent refléter la présence des espèces sensibles et si possible d'espèces indicatrices de pollution.

Le déficit d'antériorité évoqué ci-avant concernant l'ichtyofaune estuarienne ne portait pas uniquement sur la disponibilité de données d'inventaires mais aussi sur l'expertise scientifique et les recherches sur la mise au point d'indicateurs d'états écologique opérationnels. Sans données, ce travail de recherches et d'expertise n'avait naturellement pas été mené à bien au préalable. L'un des atouts majeurs de la coordination menée par l'Irstea est 
venu de la forte implication de cet institut sur des thématiques similaires pour les eaux continentales (développement d'indicateurs «poissons » en rivière et en lac; Oberdorff et al., 2002; Pont et al., 2007; Roset et al., 2007). La forte antériorité d'expertise sur les cours d'eau (plusieurs décennies) et l'implication de l'Irstea dans celle-ci a pu être mise à profit pour prendre de bonnes options stratégiques et gagner du temps (qui manquait cruellement, les échéances étant très serrées, sans données jusqu’en 2007 pour produire un état en 2013 ; le temps de la science n'est pas le temps des gestionnaires ou des politiques!).

Sur la base de cette expertise et en suivant aussi les préconisations de la DCE, une démarche de type DPSIR (Driving Forces, Pressures, State, Impacts, Response; Borja et al., 2006) a été adoptée. Cette démarche générale vise à :

- identifier les sources de pressions anthropiques (Driving forces, D) et à quantifier ces pressions (Pressures, P),

- mesurer l'état (State, S) à partir d'indicateurs, en l'occurrence mesurer l'état écologique des estuaires à partir d'indicateurs basés sur l'ichtyofaune,

- mesurer l'effet des pressions sur l'ichtyofaune (Impact, I),

- prendre des mesures (Réponse, R) pour limiter et réduire les pressions en cause si l'état n'est pas bon.

Pour faire simple, dans le cadre de la mise en œuvre de la DCE cette démarche permet, si le bon état écologique n'est pas atteint, que la Réponse ( $\mathrm{R}$, les mesures pour atteindre le bon état) vise le contrôle des Forces motrices et des Pressions (DP) afin d'améliorer l'État (S) en limitant les Impacts (I). Cette démarche peut a priori paraitre un peu virtuelle et théorique présentée de la sorte. Toutefois, elle est apparue et s'est imposée, grâce à l'éclairage d'experts de l'ichtyofaune des eaux douces au recul conséquent (e.g. Didier Pont, Irstea), comme la seule à même d'orienter les actions des gestionnaires dans leur objectif d'amélioration de la qualité écologique des masses d'eau et donc de répondre aux objectifs de la DCE. L'état écologique est ainsi mesuré par un écart à une référence, correspondant à une masse d'eau très peu ou pas impactée par les activités humaines, à partir d'une relation liant l'état écologique au niveau de pression s'exerçant sur les écosystèmes. En effet si l'état écologique n'est pas qualifié en fonction de l'impact des pressions subies, il paraît difficile, lorsque le bon état n'est pas atteint, de prendre des mesures pour parvenir à améliorer cet état en diminuant les pressions, ce qui correspond aux objectifs de la DCE.

Ce cadrage a permis de développer des indicateurs en suivant une démarche DPSIR. Beaucoup de travaux de recherche avaient permis de mesurer, par des approches expérimentales (Gilliers et al., 2012) ou des travaux de laboratoire basés sur des prélèvements in situ (Gilliers et al., 2006), les conséquences de la dégradation de la qualité des eaux et des habitats estuariens sur différentes espèces de l'ichtyofaune à des stades variables de leur cycle de vie. Les effets néfastes et donc l'impact des pressions sur la croissance et la survie des poissons ainsi que sur leur capacité de reproduction avaient été clairement mis en évidence (cf. reviews de Peterson, 2003 et Vasconcelos et al., 2014) par des approches portant sur une ou quelques espèces. Toutefois la nécessité de réaliser un diagnostic (i) à des coûts humains et financiers raisonnables, (ii) sur un grand nombre de masses d'eau échantillonnées à deux saisons par année et au minimum trois années de suite (iii) portant sur un large panel d'espèces en intégrant des notions d'abondance et de diversité, a réduit le panel des approches susceptibles d'être mises en œuvre à des analyses des données collectées in situ. Il n'était en effet pas réaliste d'effectuer sur les échantillons collectés, des analyses de laboratoire approfondies pour qualifier la croissance, la mortalité ou l'état physiologique d'un panel d'espèces représentatif de façon exhaustive et récurrente. Le travail s'est donc focalisé sur des indicateurs portant sur l'abondance et la richesse spécifique de l'ichtyofaune. Du fait de la répartition biogéographique des différentes espèces, à l'origine de fortes spécificités régionales, et du nombre important d'espèces recensées (297 espèces ont été identifiées au cours de l'échantillonnage des masses d'eau de transition en France métropolitaine entre 2005 et 2012); il n'était pas possible de se focaliser sur l'échelle spécifique pour qualifier les abondances. Pour aller plus loin qu'une simple abondance globale de poissons, et la richesse spécifique associée, les espèces ont été ventilées en guildes décrivant leur utilisation des estuaires, leur régime alimentaire et leur position dans la colonne d'eau. Cette approche qui se base sur la diversité fonctionnelle des peuplements 
de poissons est fréquemment utilisée pour ce type d'analyse intégrative (Nicolas et al., 2010b). Elle permet de comparer des systèmes éloignés dont la composition spécifique des peuplements est très différente, notamment du fait de la succession d'espèces sur un gradient latitudinal et thermique, et autorise une description assez complète des fonctions écologiques avec un nombre limité de métrique (Delpech et al., 2010).

Le but de l'analyse était donc de décrire, à l'aide de modèles statistiques, la variation de l'abondance et de la richesse spécifique au sein de ces différentes guildes en fonction du niveau de pressions anthropiques, tout en tenant compte des autres sources de variabilité : différences intrinsèques entre les façades (Méditerranée, Atlantique et Manche-Mer du Nord), différences entre les grands et les petits estuaires (intégrant aussi l'effet de l'engin de prélèvement, spécifiques à chacun de ces deux types de masses d'eau), gamme de salinité et saisonnalité. Ensuite, les métriques qui répondent aux pressions anthropiques peuvent être utilisées pour développer des indicateurs et fixer des normes. La conjonction de la note donnée par chacun de ses indicateurs permet enfin d'établir un état écologique sur la base des peuplements de poissons (figure 2).

Une première phase du travail a porté uniquement sur la guilde des poissons juvéniles marins c'est-àdire des espèces marines fortement dépendantes des estuaires au stade juvénile. Cette dépendance concerne notamment des ressources importantes en terme d'exploitation puisque $44 \%$ des espèces d'intérêt halieutique faisant l'objet d'une évaluation en Atlantique Nord présentent une dépendance côtière et/ou estuarienne à un moment donné de leur cycle de vie, ces espèces représentant $77 \%$ du tonnage des débarquements des espèces évaluées (Seitz et al., 2014). L'analyse a porté sur la réponse, en terme d'abondance et de richesse spécifique, de ces juvéniles d'espèces marines aux sources naturelles de variabilité et au niveau de contamination chimique des estuaires (Courrat et al., 2009). Ce travail réalisé à l'aide de modèles linéaires généralisés a permis de démontrer que si la variabilité liée aux facteurs naturels est forte, il est néanmoins possible de discriminer les milieux contaminés au sein desquels les densités de ces poissons juvéniles sont significativement plus faibles (figure 3). Une

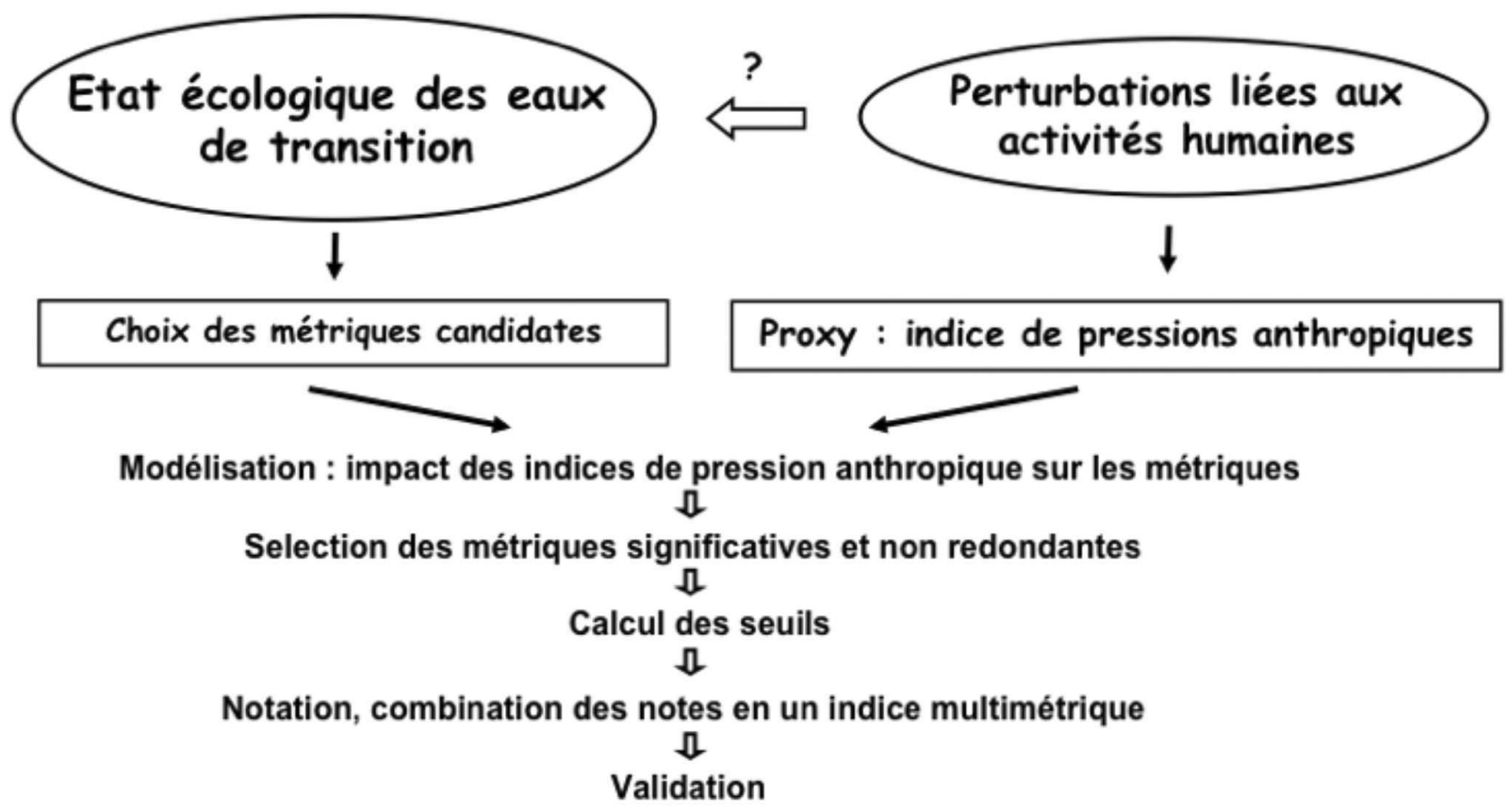

Figure 2 : Protocole d'analyse des données d'ichtyofaune pour développer un indicateur d'état écologique des estuaires (d'après Delpech et al., 2010)

Methodology based on fish fauna data for building an ecological quality index of estuaries (adapted from Delpech et al., 2010) 


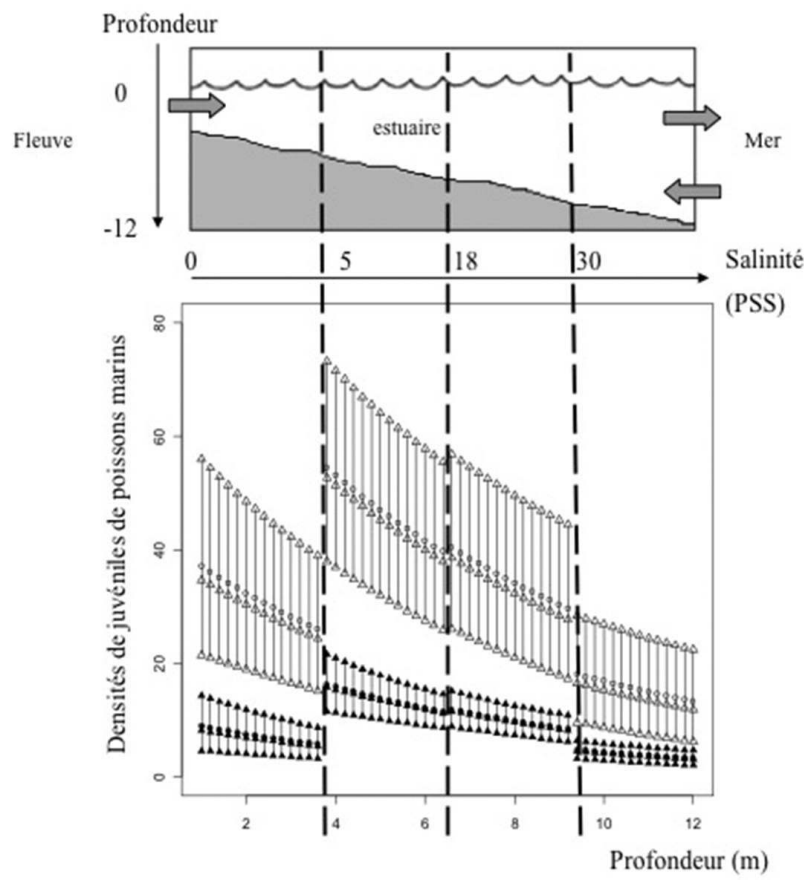

Figure 3 : Estimation des densités automnales de juvéniles de poisons marins (valeur médiane et intervalle de confiance $[10,90] \%$ ) dans les grands estuaires de la côte Atlantique, le long d'un gradient amont-aval de salinité et de profondeur selon le degré de contamination chimique (triangles pleins, contamination chimique forte; triangles vides, contamination faible à très faible) (d'après Courrat et al., 2009)

Autumnal estimated densities of young marine fishes (median value and quantiles $[10,90] \%)$ in large estuaries of the Atlantic coast, along an upstream-downstream gradient in salinity and depth depending on level of chemical contamination (solid triangles: strong chemical contamination; open triangles: low to very low contamination ) (from Courrat et al., 2009)

même tendance a été constatée en ce qui concerne leur richesse spécifique mais sans que le niveau de discrimination soit aussi marqué. Ce travail a aussi conduit à préciser le protocole d'échantillonnage de l'ichtyofaune en permettant d'estimer que le niveau de fiabilité de l'estimation est satisfaisant lorsque l'on dispose d'au minimum 8 traits par gamme de salinité.

Cette première étape réussie, la démarche a été généralisée à l'ensemble des guildes décrivant l'ichtyofaune (utilisation des estuaires, régime alimentaire et position dans la colonne d'eau). Douze métriques présentaient une réponse significative aux pressions anthropiques, liées à une moindre abondance et à une diversité spécifique réduite pour une contamination chimique plus forte. Un travail de sélection s'est opéré afin de ne conserver que les métriques présentant un effet discriminant (les inter- valles de confiance entre secteurs contaminés et non contaminés ne doivent pas se recouper (figure 4) pour éviter des confusions sur le diagnostic). Puis certaines métriques discriminantes n'ont pas été retenues parce qu'elles étaient redondantes (e.g. les poissons benthiques (position dans la colonne d'eau) et les poissons qui mangent des invertébrés benthiques (régime alimentaire) sont en très grande partie constitués des mêmes espèces). Au final, sept métriques discriminantes et non redondantes ont été sélectionnées : la densité totale de poissons, la densité de poissons migrateurs amphihalins, la densité de poissons marins juvéniles (dans les zones polyhaline et mésohaline), la densité des poissons d'eau douce (dans la zone oligohaline et en eau douce soumise à marée), la densité des espèces résidentes estuariennes, la densité d'espèces benthiques et la richesse taxonomique totale (Delpech et al., 2011). Des approches statistiques plus poussées, utilisant des méthodes bayésiennes appropriées pour prendre en compte l'incertitude et les différentes sources de variabilité ont ensuite été utilisées pour valider ces indicateurs et l'adéquation entre le protocole de collecte de données et le niveau de fiabilité requis. Ce travail a aussi fait l'objet d'une validation en intégrant la connaissance experte des spécialistes de l'ichtyofaune des masses d'eau estuariennes, en tenant compte d'autres sources de pressions anthropiques (Tableau et al., 2013).

Au final, on dispose donc de grilles d'évaluation de la qualité des eaux qui permettent de donner une note de qualité pour chaque métrique retenue (tableau 1), par classe de salinité au cours de chaque campagne (échantillonnage d'une masse d'eau au cours d'une saison). Le calcul des seuils et l'attribution d'un score à chaque classe de qualité se fait en fonction du recouvrement ou non des intervalles de confiance obtenues pour chaque métrique vis-à-vis d'un certain niveau de contamination ou d'anthropisation (figure 4).

Il s'agit ensuite de conjuguer ces notes pour obtenir une qualification de l'état écologique à partir de l'indicateur global calculé sur les données d'ichtyofaune, nommé ELFI (Estuarine and Lagoon Fish Index; Delpech et al., 2010). Pour chaque métrique, une note est attribuée à chaque classe de salinité pour laquelle plus de 8 traits de chalut ont été réalisés, au cours d'une campagne. On obtient la note pour une masse d'eau, en procédant à la moyenne des notes obtenues dans chaque classe de salinité 


\begin{tabular}{cllcccc} 
Ecoregion & Saison & $\begin{array}{c}\text { Classe de } \\
\text { salinité }\end{array}$ & Seuil Me-Ma & Seuil Mo-Me & Seuil B-Mo & Seuil TB-B \\
\hline ATL & automne & 1-oligo & 0,54 & 1,1 & 1,7 & 2,3 \\
ATL & automne & 2-méso & 1,2 & 1,8 & 2,4 & 3 \\
ATL & automne & 3-poly & 0,89 & 1,5 & 2,1 & 2,7 \\
ATL & printemps & 1-oligo & 0,54 & 1,1 & 1,7 & 2,3 \\
ATL & printemps & 2-méso & 1,2 & 1,8 & 2,4 & 3 \\
ATL & printemps & 3-poly & 0,89 & 1,5 & 2,1 & 2,7 \\
MAN & automne & 1-oligo & 0,54 & 1,1 & 1,7 & 2,3 \\
MAN & automne & 2-méso & 1,2 & 1,8 & 2,4 & 3 \\
MAN & automne & 3-poly & 0,89 & 1,5 & 2,1 & 2,7 \\
MAN & printemps & 1-oligo & 0,54 & 1,1 & 1,7 & 2,3 \\
MAN & printemps & 2-méso & 1,2 & 1,8 & 2,4 & 3 \\
MAN & printemps & 3-poly & 0,89 & 1,5 & 2,1 & 2,7
\end{tabular}

Tableau 1 : Exemple de valeur de seuils en 5 classes (Très Bon : TB, Bon : B, Moyen : Mo, Médiocre : Me, et Mauvais : Ma) pour la métrique « Densité d'espèces benthiques »

Les valeurs affichées correspondent à une densité moyenne sur l'ensemble des traits de chalut réalisés pour chaque classe de salinité (1-oligo : 5 PSS, 2-mesohalin : ]5,18] PSS, 3-poly : > 18PSS) au printemps ou en automne, par écorégion (ATL : Atlantique; MAN : Manche), dans le cas des grands estuaires.

Example of thresholds value through 5 classes (Very Good: TB, Good: B, Middle: Mo, Poor: Me and Bad: Ma) for the metric "density of benthic species"

The values represent an average density for all trawl tows performed for each salinity class (1-oligo: $\leq 5$ PSS, 2-mesohalin:] 5.18] PSS, 3-poly:> 18PSS) during spring or autumn, by Ecoregion (ATL: Atlantic; MAN: Manche), for large estuaries.

Figure 4 : Système de scoring utilisé pour les métriques

a : système classique en 3 classes en l'absence de chevauchement des intervalles de confiance. b : système adapté en 5 classes lorsque le chevauchement des 3 classes est limité. c : chevauchement trop important, impossibilité de définir des seuils robustes, métrique non retenue. d : système en 5 classes sans chevauchement des intervalles de confiance.

metric

Scoring system used for

a: conventional system into 3 classes with non-overlapping quantiles. $b$ : methodology adapted into 5 classes with a slightly overlap of the 3 classes. $c$ : too strong overlapping, leading to reject the metric. $d$ : system into 5 classes with non-overlapping quantiles.

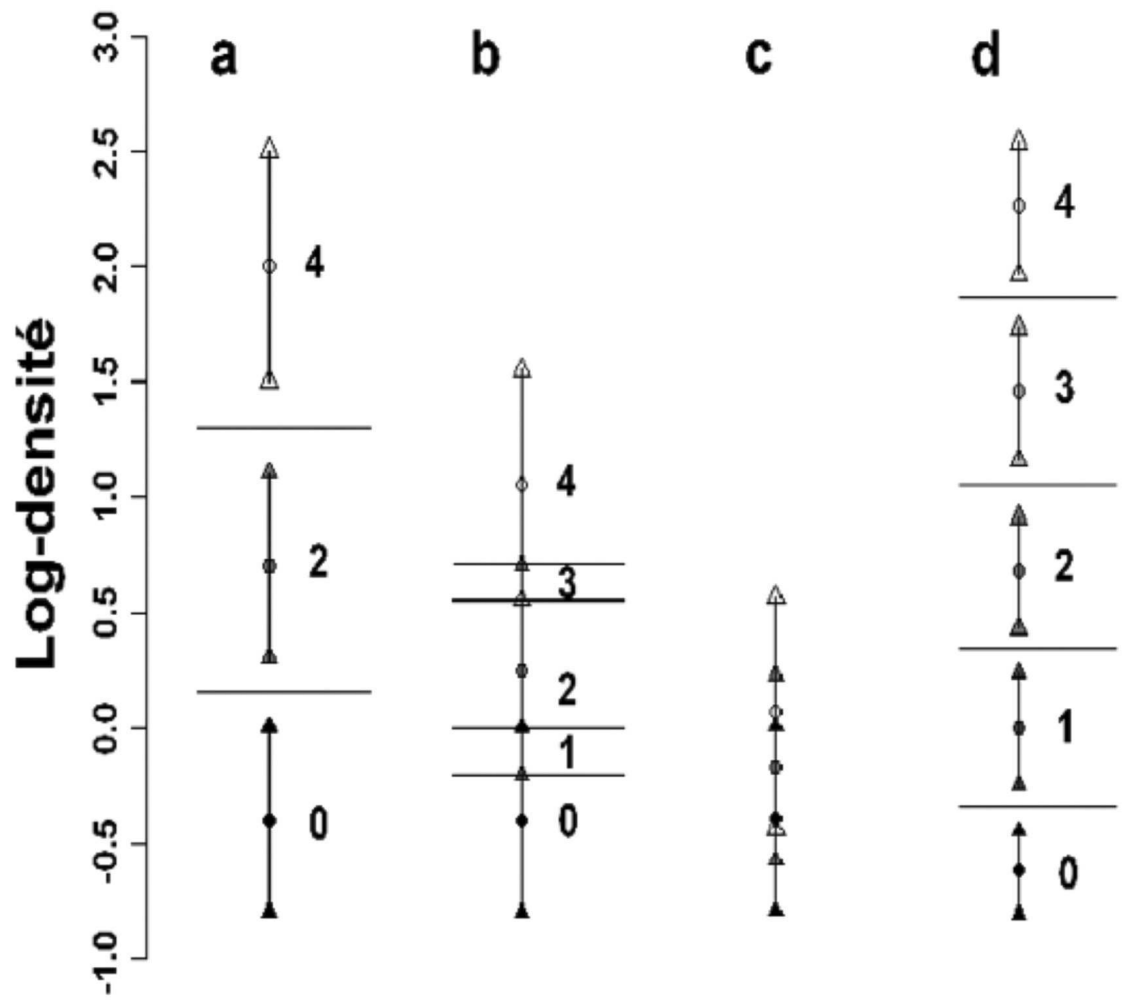


(note entre 0 et 1 , la valeur 1 étant la note maximale). Dans le cadre de la DCE, on procède à deux campagnes par année (printemps et automne) pendant trois années successives pour pondérer les évènements extrêmes. L'indicateur ELFI est basé sur la moyenne des notes (une note pour chacune des sept métriques, sur deux saisons au cours de trois années) obtenues sur une période de trois ans (tableau 2).

Une démarche de même type a permis de développer des indicateurs pour les lagunes (Drouineau et al., 2012), en intégrant quatre métrique dont deux portant sur l'abondance et deux autres sur la diversité spécifique de différentes guildes.

Ainsi, il est possible de qualifier l'état écologique des masses d'eau de transition à partir de l'ichtyofaune sur la base d'indicateurs statistique- ment fiables basés sur les données des campagnes d'échantillonnage dévolues à cette estimation.

\section{UNE HARMONISATION A POSTERIORI À L'ÉCHELLE EUROPÉENNE}

Chaque pays européen a suivi une démarche de même type afin de mettre en place un réseau de surveillance de l'ichtyofaune et de développer des indicateurs de qualité écologique basés sur ces données de surveillance. Les méthodes d'échantillonnage varient beaucoup selon les pays (Nicolas et al., 2010a), tout comme les indicateurs retenus (Perez Dominguez et al., 2012; Pasquaud et al., 2013).

Un exercice d'inter-étalonnage de huit indicateurs «poissons » utilisés en Atlantique, Manche et Mer du Nord (Portugal, Espagne, France, Belgique,

\begin{tabular}{|c|c|c|c|c|c|c|}
\hline Agence & Nom masse eau de transition & ELFI2009 & ELFI2010 & ELFI2011 & ELFI 2012 & $\begin{array}{c}\text { Note rapportage } \\
\text { période 2009-2011 }\end{array}$ \\
\hline AG & Adour amont & 0,38 & 0,31 & 0,13 & & 0,273 \\
\hline AG & Adour aval & 0,41 & 0,3 & 0,18 & & 0,297 \\
\hline SN & Baie des Veys & & 0,9 & 0,31 & 0,48 & 0,563 \\
\hline AG & Bidassoa & 0,43 & 0,04 & 0,36 & & 0,277 \\
\hline LB & Blavet & 0,86 & 0,96 & 0,93 & & 0,917 \\
\hline AG & Charente & 0,67 & 0,72 & 0,79 & & 0,727 \\
\hline AG & Dordogne fluvial & 0,36 & 0,65 & 0,46 & & 0,490 \\
\hline SN & Fond de baie du Mont Saint-Michel & & 0,92 & 0,98 & 1 & 0,967 \\
\hline AG & Garonne fluvial amont & 0,36 & 0,44 & 0,27 & & 0,357 \\
\hline AG & Gironde aval & 0,67 & 0,44 & 0,5 & & 0,537 \\
\hline AG & Gironde centrale & 0,19 & 0,23 & 0,34 & & 0,253 \\
\hline RMC & Grand Rhône & & 0,79 & 0,5 & & 0,645 \\
\hline LB & Laïta & 0,5 & 0,72 & 0,48 & & 0,567 \\
\hline LB & Loire & 0,51 & 0,49 & 0,41 & & 0,470 \\
\hline LB & Morlaix & 0,73 & 0,59 & 0,54 & & 0,620 \\
\hline SN & Orne & & 0,63 & 0,38 & 0,82 & 0,610 \\
\hline SN & Risle & & 0,63 & 0,49 & 0,79 & 0,637 \\
\hline SN & Seine amont & & 0,04 & 0,19 & 0,08 & 0,103 \\
\hline $\mathrm{SN}$ & Seine aval & & 0,38 & 0,3 & 0,39 & 0,357 \\
\hline SN & Seine central & & 0,06 & 0,06 & & 0,060 \\
\hline AG & Seudre & 0,46 & 0,5 & 0,61 & & 0,523 \\
\hline LB & Sèvre Niortaise & 0,63 & 0,59 & 0,5 & & 0,573 \\
\hline AP & Somme & 0,94 & & & & 0,940 \\
\hline LB & Vilaine & 1 & 0,9 & 0,92 & & 0,940 \\
\hline
\end{tabular}

Tableau 2 : Calcul de l'indicateur ELFI (code Agence de l'eau : LB Loire Bretagne, AG Adour Garonne, SN Seine Normandie, AP Artois Picardie et RMC Rhône Méditerranée et Corse. Vide = année sans échantillonnage)

Les seuils des classes de qualité sont : Très bon=0,91; Bon $=0,675 ;$ Moyen $=0,45$; Médiocre $=0,225$; et Mauvais $<0,225$. Calculation of the ELFI indicator (Water Agency code. LB Loire Bretagne, AG Adour Garonne, SN Seine Normandy, AP Picardy and Artois RMC Rhone Mediterranean and Corsica Empty = year without sampling)

The thresholds separating quality classes are: Very good =0.91; Good =0.675; Average =0.45; Poor =0.225; and Bad $<0.225$. 
Pays-Bas, Allemagne, Royaume Uni et Irlande) a été conduit entre 2006 et 2012. Ce travail d'inter-étalonnage (Delpech et al., 2011) a permis de vérifier que les approches mises en place par les différents pays conduisent à des évaluations similaires de l'état écologique. L'Union européenne, en concertation avec le Joint Research Centre (JRC, le service scientifique de l'UE) basé à Ispra en Italie, a validé le travail réalisé par les experts des différents États membres et les indicateurs «poissons » qui ont été intégré dans l'exercice ont tous été validés pour la région géographique Nord-Est Atlantique. Pour la Méditerranée, ce travail d'inter-étalonnage reste encore à terminer. Seul la Grèce, l'Italie et la France ont développé des outils de classification mais des développements restent encore à prévoir.

\section{Conclusion et nOuVElles perspectives, la Directive Cadre Stratégie pour le Milieu Marin}

Bien que les conditions initiales défavorables en terme de données disponibles et d'expertise préalable se soient avérées particulièrement défavorables, un réseau de surveillance et des outils pour qualifier l'état écologique des estuaires et des lagunes à partir de leur ichtyofaune ont pu être développés dans les délais resserrés que fixaient la DCE (du néant en 2005 à un réseau de surveillance opérationnel en 2007 et enfin un système d'évaluation de la qualité normé et validé scientifiquement en 2010; Delpech et al., 2010). Malgré un état naturel des peuplements de poissons en estuaire ressemblant à des peuplements perturbés (estuarine quality paradox; Elliott et Quintino, 2007), il a été possible de développer une méthode fiable répondant aux objectifs de la DCE. Les données collectées ont par ailleurs contribué à améliorer les connaissances sur la biodiversité ichtyologique en estuaire (Argillier et Lepage, 2010) et à fournir de la matière à de nombreuses avancées scientifiques (e.g. Nicolas et al., 2010a,b), contribuant à combler une partie du retard sur les avancées réalisées sur l'ichtyofaune d'eau douce.

Sur la base du constat réalisé à l'issue du premier plan de gestion, en 2013, le travail va se poursuivre.
Il va notamment être nécessaire de prendre des mesures pour améliorer l'état écologique des masses d'eau pour lesquelles l'ichtyofaune ne conduit pas à un diagnostic satisfaisant (volet réponse $\mathrm{R}$ de la démarche DPSIR). L'expertise acquise depuis le début du processus par les acteurs en charge de la collecte des données sur ces différentes masses d'eau va être très utile dans ce cadre. Pour ces masses d'eau, Il va s'agir de raffiner spatialement le diagnostic global (e.g. le mauvais état de l'ichtyofaune peut il être imputé à une section particulière d'un estuaire ?), puis de mettre en évidence les pressions anthropiques locales susceptibles de cette dégradation. Cette expertise permettra ensuite aux acteurs en charge de la gestion locale de ces masses d'eau de transition de mettre en œuvre des mesures pour parvenir à améliorer cette état, conformément à la DCE.

La directive cadre européenne stratégie pour le milieu marin (DCSMM), émise en 2008, s'inscrit dans la suite de la DCE, en conformité avec les résolutions de Johannesburg (2002). Elle s'apparente à une extension de la DCE à l'ensemble de l'espace maritime européen. En effet, son objectif premier est de prendre toutes les mesures nécessaires pour réaliser ou maintenir un bon état écologique du milieu marin au plus tard en 2020. Chaque état membre élabore sa propre stratégie en tenant compte de l'échéancier imposé par la directive (2012 : évaluation et description de l'état de l'environnement, définition du bon état écologique des océans, création des cibles et points de référence; 2014 : établissement du programme de surveillance; 2015-2016 : établissement puis mise en œuvre du programme de mesures; 2020 : atteinte du bon état écologique). Le bon état écologique est défini au travers de 11 descripteurs qualitatifs énoncés dans la directive. Parmi ces descripteurs, le premier (Diversité biologique) et le quatrième (Réseau trophique) font notamment appel à des estimations de la qualité écologique portant sur des descripteurs de l'ichtyofaune. Une démarche similaire est donc désormais en cours en milieu marin (Henriques et al., 2014). 


\section{Remerciements}

Nous souhaitons remercier chaleureusement toutes les personnes impliquées dans cette course contre la montre depuis une dizaine d'années, et notamment Christine Delpech, Anne Courrat, Stéphanie Pasquaud, Hilaire Dronineau, Michel Girardin, Adrien Tableau, Vincent Bouju et Didier Pont à l'Irstea, ainsi que le très large panel de personnes impliquées dans la collecte des données de terrain au sein de nombreux organismes de recherche et d'enseignement supérieur et de bureaux d'études. Nous souhaitons également remercier les organismes financeurs, Agence de l'eau Adour-Garonne, Agence de l'eau Loire- Bretagne, Agence de l'eau Seine-Normandie, Agence de l'eau Artois-Picardie, Agence de l'eau Rhône Méditerranée et Corse, Onema et programme LITEAU (ministère en charge du développement durable) qui ont permis la mise en place d'un réseau de surveillance et le développement de l'indicateur ELFI. Merci aussi à Grégoire Maillet, éditeur de la session, ainsi qu'aux deux relecteurs anonymes pour leurs suggestions constructives

\section{Bibliographie}

Argillier C., Lepage M. 2010. Que peut-on attendre de la directive cadre européenne sur l'eau en matière de bioviversité ? Sciences Eaux \& Territoires, 3, p. 84-87.

Borja A., Galparsoro I., Solaun O., Muxika I., Tello E.M., Uriarte A., Valencia V., 2006. The european water framework directive and the DPSIR, a methodological approach to assess the risk of failing to achieve a good ecological status, Estuarine, Coastal and Shelf Science, 66, p. $4-96$

Claisse D., 1989. Chemical contamination of French coasts. The results of a ten years Mussel watch, Marine Pollution Bulletin, 20, p. 523-528.

Courrat A., Lepage M., Girardin M., Laffargue P., Nicolas D., Lobry J., Le Pape O. 2009. Anthropogenic disturbance on nursery function of estuarine areas for marine species, Estuarine, Coastal and Shelf Science, 81(2), p. 179-190.

Delpech C., Courrat A., Pasquaud S., Lobry J., Le Pape O., Nicolas D., Girardin M., Bö̈t P., Lepage M., 2010. Development of a fish-based index to assess the ecological quality of transitional waters: The case of French estuaries, Marine Pollution Bulletin, 60, p. 908-918.

Delpech C., Drouineau H., Lepage M., 2011 . Amélioration de la robustesse de l'indicateur ELFI et état d'avancement de l'intercalibration européenne. Convention de partenariat ONEMA-Cemagref 2010, Cemagref Groupement de Bordeaux, France, 98 p.
Drouineau H., Lobry. J., Delpech C., Bouchoucha M., Mahévas S., Courrat A., Pasquaud S., Lepage M., 2012. A bayesian framework to objectively combine metrics when developin stressor specific multimetric indicators, Ecological Indicators, 13, p. 314-21.

Elliot M., Quintino V., 2007. The estuarine quality paradox, Environmental homéostasis and the difficulty of detecting anthropogenic stress in naturally stressed areas, Marine Pollution Bulletin, 54, p. 640-645.

Gilliers C., Claireaux G., Galois R., Loizeau V., Le Pape O., 2012. Influence of hydrocarbons exposure on survival, growth and condition of juvenile flatfish: a mesocosm experiment, Journal of Life Science, 4(2), p. 113-122.

Gilliers C., Le Pape O., Morin J., Desaunay Y., Amara R., 2006. Are growth and density quantitative estimators of essential fish habitat quality? An application to the common sole Solea solea nursery grounds, Estuarine Coastal and Shelf Science, 69, p. 96-106.

Henriques S., Pais M., Vasconcelos R., Murta A., Azevedo M., Costa M., Cabral H., 2014. Structural and functional trends indicate fishing pressure on marine fish assemblages, Journal of Applied Ecology, 51(3), p. 623-631.

Lepage M., Girardin M., 2006. Inventaire Poisson dans les eaux de transition. Protocole d'échantillonnage pour le District Rhône Méditerranée et Corse. Rapport Irstea, Cestas France, $32 \mathrm{p}$.

Lobry J., Mourand L., Rochard E., Elie P., 2003. Structure of the Gironde estuarine fish assemblages: a comparison of European estuaries perspective, Aquatic Living Resources, 16, p. 47-58.

Nicolas D., Lobry J., Lepage M., Sautour B., Le Pape O., Cabral H., Uriarte A., Boët P., 2010a. Fish under influence: a macroecological analysis of relations between fish species richness and environmental gradients among European tidal estuaries, Estuarine, Coastal and Shelf Science, 86, p. 137-147.

Nicolas D., Lobry J., Le Pape O., Bö̈t P., 2010b. Functional diversity in European estuaries: Relating the composition of fish assemblages to the abiotic environment, Estuarine, Coastal and Shelf Science, 88, p. 329-338.

Oberdorff T, Pont D., Hugueny B., Porcher J.P., 2002. Development and validation of a fish-based index for the assessment of "river health" in France, Freshwater Biology, 47, p. 1720-1734.

Pasquaud S., Courrat A., Fonseca V., Gamito R., Gonçalves C., Lobry J., Lepage M., Costa M., Cabral H., 2013. Strength and time lag of relationships between human pressures and fish-based metrics used to assess ecological quality of estuarine systems, Estuarine, Coastal and Shelf Science, 134, p. 119-127.

Perez-Dominguez R., Maci S., Courrat A., Lepage M., Borja A., Uriarte A., Neto J., Cabral H., St Raykov V., Franco A., Alvarez M.C., Elliott M., 2012. Current developments on fish-based indices to assess ecological-quality status of estuaires and lagoons, Ecological Indicators, 23, p. 34-45. 
Peterson M., 2003. A conceptual view of environment-habitat-production linkages in tidal river estuaries, Reviews in Fisheries Science, 11(4), p. 291-313.

Pont D., Hugueny B., Rogers C., 2007. Development of a fish-based index for the assessment of river health in Europe: the European Fish Index, Fisheries Management and Ecology, 14, p. 427-439.

Roset N., Grenoullet G., Goffaux D., Pont D., KesteMEONT P., 2007. A review of existing fish assemblage indicators and methodologies, Fisheries Management and Ecology, 14 (6), p. 393-405.

Seitz R.D., Wennhage H., Bergström U., Lipcius R.N., YseBAERT T., 2014. Ecological value of coastal habitats for com- mercially and ecologically important species, ICES Journal of Marine Science, 71 (3), p. 648-665.

Tableau A., Drouineau H., Delpech C., Pierre M., Lobry J., Le Pape O., Breine J., Lepage M., 2013. A fish-based index of estuarine ecological quality incorporating information from both traditional scientific fish survey and experts knowledge, Ecological Indicators, 32, p. 147-156.

Vasconcelos R., Eggleston D.B., Le Pape O., Tulp I., 2014. Patterns and processes of habitat-specific demographic variability in exploited marine species, ICES Journal of Marine Science, 71, p. 638-647. 